\title{
Der Mensch zwischen Fragezeichen und Ausrufezeichen
}

\author{
Zu einer Pensée von Blaise Pascal (1623-1662)
}

Pierre Bühler

Jeder Mensch ist ein Abgrund; es schwindelt einem, wenn man hinabsieht.

Georg Büchner, Woyzeck

De profundis clamavi ad te Domine

Psalm 130,1

\section{Einstieg}

Cognitio Dei et hominis sei das Grundthema der Theologie, betonten die Reformatoren. ${ }^{1}$ Für die Einlösung dieser Aufgabe gibt es in der Theologiegeschichte mehr oder weniger ruhige, behagliche Ansätze und Ansätze, die diese Aufgabe nur mit «Furcht und Zittern» angehen können. Zur ersten Sorte gehören etwa die theologischen Summen des Mittelalters, so zum Beispiel die von Thomas von Aquin, in der mit grösster Sorgfalt, aber auch in aller Ruhe die menschliche Wirklichkeit in allen Einzelheiten durchforscht wird, um in ihr den Gottesbezug aufzuspüren. Zur zweiten Sorte zählt Kierkegaards Religionsphilosophie, in der die Gottesfrage nicht ohne Angsterfahrung zu denken ist, oder die dialektische Theologie mit ihrer Betonung der Krise im Zeichen der Begegnung mit dem ganz Anderen.

Freilich besteht zwischen diesen zwei Einstellungen keine radikale Alternative. Trotzdem aber ist die Akzentsetzung nicht gleichgültig. An ihr misst sich eine Art Grundstimmung in Hinsicht auf die Verknüpfung von Anthropologie und Theologie: Gehört die Gottesbeziehung zur selbstverständlichen, gewöhnlichen Ausstattung des Menschen oder wird sie dort erst recht virulent, wo der Mensch sich selbst in seinem Menschsein zur Frage wird?

1 Vgl. G. Ebeling, Cognitio Dei et hominis, in: ders., Lutherstudien I, Tübingen 1971, 221-272 (bei Luther:WA 40, 2; 327, 11-328, 2). 
Wenn wir uns im Folgenden mit Pascal befassen², so muss gleich betont werden, dass seine Anthropologie eher zur zweiten Sorte der oben unterschiedenen Ansätze gehört. Das soll hier anhand einer für unsere Thematik interessanten Stelle erläutert werden, einer Pensée, die Pascal selbst in ein Bündel zum Thema «Contrariétés» eingeordnet hatte. ${ }^{3}$ 〈Contrariété» heisst auf Französisch «Gegensätzlichkeit〉, 〈Widerspruch〉, 〈Widerstreit〉, aber auch 〈Widerwärtigkeit〉, 〈Unannehmlichkeit). Es handelt sich um die Pensée 131. ${ }^{4}$ Sie ist bis in ihre Form hinein ein sehr «unruhiger» Text: Unzufrieden mit sich selbst hat Pascal seinen Text mehrmals durchgestrichen und seinen Gedankengang in immer neuen Ansätzen umformuliert.

\section{Gegensätzlichkeiten}

Dass der Mensch in seinem Wesen durch spannungsvolle Gegensätzlichkeiten konstituiert ist und deshalb immer im Widerstreit mit sich selbst lebt, das gehört bei Pascal zum Grundtenor seiner Anthropologie. Ganz prägnant wird das in den Pensées durch die Spannung von Grösse und Elend zum Ausdruck gebracht, die Pascal in vielen Variationen durchspielt. Deshalb gilt sozusagen als Motto seiner Anthropologie (wobei unklar ist, ob das Ich, das hier spricht, das Ich des Philosophen oder das göttliche Ich ist) :

Wenn er sich rühmt, demütige ich ihn.

Wenn er sich demütigt, rühme ich ihn.

Und ich widerspreche ihm immer.

Bis er begreift,

dass er ein unbegreifliches Monstrum ist (86).

Interessant sind an dieser Stelle, die unmittelbar vor unserer Pensée steht, zwei Aspekte: Einerseits geschieht eine Verdoppelung des Widersprechens: Dem Menschen soll in seinen Widersprüchlichkeiten immer wieder widersprochen werden, so dass er nie zur Ruhe kommt, weder beim Elend noch bei der Grösse, sondern stets Elend in der

\footnotetext{
2 Alois Rust und ich haben im vergangenen Frühjahrssemester ein interdisziplinäres Seminar über Pascals Pensées veranstaltet.

3 Ich zitiere die Pensées nach: B. Pascal, Gedanken über die Religion und einige andere Themen. Hg. v.J.-R. Armogathe. Aus dem Französ. übersetzt von U. Kunzmann (Universal-Bibliothek, Nr. 1622), Stuttgart 1997 (Leipzig 1987). Diese Ausgabe zählt die Gedanken nach der Zählung von L. Lafuma (vgl. B. Pascal, Pensées. Texte établi par L. Lafuma [Essais, n 94], Paris 1962).

4 In der Ausgabe von J.-R. Armogathe: 87-93, hier vor allem 89-92. Die Seitenzahlen in Klammern beziehen sich auf diese Ausgabe.
} 
Grösse und Grösse im Elend wahrnimmt. Andererseits verbindet Pascal damit eine Paradoxierung des angestrebten Ziels: Mit dieser «Verfolgung» des Menschen durch seine Widersprüche hindurch soll er dazu angehalten werden, sich als unbegreifliches Monstrum zu begreifen. Wie hängt dieses anthropologische Programm mit der Theologie zusammen? Das wollen wir in der Pensée 131 genauer beobachten.

\section{Zwischen dem Pyrrhonismus und dem Dogmatismus}

Pascal setzt bei einer erkenntnistheoretischen Debatte ein, die er als Widerstreit zwischen den Pyrrhoniker und den Dogmatisten kennzeichnet. ${ }^{5}$ Dabei geht es um die Grundfrage, ob der Mensch in Hinsicht auf die Grundprinzipien, seine Herkunft und sein Ziel, Anfang und Ende des Universums usw. zu einer gewissen Erkenntnis gelangen kann. In dieser Frage entsteht eine Art Pattsituation, die Pascal als "offene[n] Krieg» bezeichnet (89): Es stehen einander zwei Positionen gegenüber, von denen keine wirklich zu gewinnen vermag. Der Pyrrhonismus ist ein empirischer Skeptizismus: Die Vernunft vermag es nicht, über ein reines Fühlen hinauszukommen, und dieses Fühlen kann keinen Beweis erbringen, so dass der Mensch in Hinsicht auf die Grundüberzeugungen eigentlich nur durch Glauben zur Gewissheit gelangen kann. Demgegenüber steht der Dogmatismus, den Pascal hier in einer kartesianisch anmutenden Begrifflichkeit zum Ausdruck bringt: Es ist der Vernunft durch Natur eingegeben, dass sie Wahrheit und Trug klar unterscheiden und deshalb auch mit ihren Mitteln eine sichere Erkenntnis der Grundprinzipien erreichen kann.

Das Problem erweist sich für Pascal auf rein epistemologischer Ebene als unlösbar, denn keine dieser zwei Positionen kann sich entscheidend gegen die andere behaupten. Deshalb kann man nicht in diesem Rahmen verweilen, wie schön das auch wäre. Die dialektische Situation des erkenntnistheoretischen Widerstreits wird zum Ort einer anthropologischen Reflexion. Die Wende von der Erkenntnistheorie zur Anthropologie wird durch folgende Frage eindeutig markiert: «Was soll also der Mensch in diesem Zustand tun?» (89) Auch hier wird wieder mit einer Gegensätzlichkeit geant-

\footnotetext{
5 An gewissen Stellen kommen noch die Akademiker hinzu; wir lassen hier der Einfachheit halber diese dritte Position beiseite, weil sie für den Hauptstrang der Argumentation nicht wesentlich ist.
} 
wortet, die zwar weiterhin epistemologisch klingt, jedoch bereits den Übergang zum Menschsein selbst vollzieht: «Soll er an allem zweifeln, soll er zweifeln, ob er wacht, ob man ihn kneift, ob man ihn verbrennt, soll er zweifeln, ob er zweifelt, soll er zweifeln, ob es ihn gibt?» (ebd.) Das kann es im Urteil Pascals für den Menschen nicht gewesen sein. Ein so radikaler Zweifel ist unvorstellbar, und Pascal folgert: « $[\mathrm{I}] \mathrm{ch}$ nehme als Tatsache an, dass es niemals einen tatsächlich vollkommenen Pyrrhoniker gegeben hat» (ebd.). Doch damit stellt sich nun die Frage, ob der Mensch den Dogmatisten Recht geben muss. Auch das erweist sich aber als unmöglich: «Soll er also im Gegenteil sagen, dass er gewiss die Wahrheit besitzt, er, der, sobald man ihn nur im geringsten drängt, dafür keinen Beweis vorbringen kann und gezwungen ist, klein beizugeben?» (ebd.)

Aus dieser doppelten Unmöglichkeit folgert nun Pascal eine Anthropologie ganz und gar mit Fragezeichen:

Welches Trugbild ist denn der Mensch? Welches noch nie dagewesene Etwas, welches Monstrum, welches Chaos, welcher Hort von Widersprüchen, welches Wunderding? Ein Richter über alle Dinge, ein schwacher Erdenwurm, ein Hüter der Wahrheit, eine Kloake der Ungewissheit und des Irrtums, Ruhm und Abschaum des Weltalls.

Wer löst diese Verwirrung? («Qui démêlera cet embrouillement?»; ebd.)

\section{Vom Fragezeichen zum Ausrufezeichen}

Diese fragende Anthropologie bleibt zunächst einmal unbeantwortet mit all ihren paradoxen Gegensätzlichkeiten: der Mensch als Richter über alle Dinge und zugleich schwacher Erdenwurm, als Hüter der Wahrheit und als Kloake der Ungewissheit und des Irrtums, als Ruhm des Weltalls und zugleich als dessen Abschaum? Die letzte Frage öffnet jedoch bereits eine andere Perspektive, als Frage nach jemandem, der diese "contrariétés» entflechten könnte, wobei anscheinend implizit mitgesetzt ist, dass es nicht der Mensch selbst sein kann.

Pascal nimmt im Folgenden den Streit zwischen den Pyrrhonikern und den Dogmatisten wieder auf, eine Stelle, die er später gestrichen hat. In der neuen Fassung schlägt der epistemologische Streit, als Streit zwischen Vernunft und Natur, ganz plötzlich um in eine in zweiter Person an den Menschen adressierte Aufforderung zur 
Selbsterkenntnis: «Erkenne also, du Hochmütiger, welches Paradoxon du für dich selbst bist. Demütige dich, ohnmächtige Vernunft!» (90)

Damit sind wir beim Ausrufezeichen angelangt. Die Aufforderung zur Selbsterkenntnis vertieft Pascal nun noch als Aufruf an die törichte Natur, wobei hier wohl die menschliche Natur zu verstehen ist. Sie soll schweigen und erfahren, "dass der Mensch unendlich über den Menschen hinausgeht» ("que l'homme passe infiniment l'homme»; ebd.). Das heisst:Was den Menschen in seinem Menschsein ausmacht, das entgeht dem Menschen, übersteigt seine Fähigkeiten. Damit wird im Nachhinein begründet, warum kurz vorher die Frage gestellt wurde, wer denn die Verwirrung lösen werde. In dieser Frage klang implizit die Gottesfrage an. Diese Frage wird nun, noch einmal vom Fragezeichen zum Ausrufezeichen schreitend, als Imperativ an die törichte Natur ausgerufen:

«[...] höre von deinem Herrn, welches deine wirkliche Lage ist, die du nicht kennst. Höre auf Gott!» (ebd.)

Mit diesem Satz ist die Paradoxie der Selbsterkenntnis wiederholt zum Ausdruck gebracht: Die wirkliche Lage wird durch die Natur nicht erkannt, bei aller Bemühung der Selbsterkenntnis. Sie wird nur erkennbar im Hören auf Gott. Deshalb kulminiert die fragende Anthropologie in diesem Imperativ mit seinem Ausrufezeichen. So steht der Mensch «zwischen den Zeichen», dem Frage- und dem Ausrufezeichen, und da allein kann er sich als den erkennen, der er wirklich ist: ein Monstrum von Grösse und Elend.

\section{Eine theologische Erläuterung der Widersprüchlichkeit}

Von dieser Stelle an ist der ganze Schlussteil der Pensée öfters durchgestrichen und umformuliert. Die Linie, die Pascal bei allem Hin und Her verfolgt, ist aber klar: Er versucht nun, theologisch zu erläutern, warum der Mensch sich in so grosser Widersprüchlichkeit erfährt, und zwar dadurch, dass er diese Widersprüchlichkeit als Spannung zwischen einer verlorenen Vollkommenheit, mit der sich eine Ahnung von Wahrheit und Glückseligkeit verbindet, und einer erlittenen Verderbnis, mit der sich Trug und Unglück über die Menschen breit macht. Dort haben, so Pascal, Grösse und Elend des Menschen ihre Quelle. Das heisst: Pascal interpretiert die wirkliche Lage («la véritable condition") des Menschen von der biblischen Urgeschichte 
her als die bleibende Spannung zwischen der geschöpflichen Würde des Menschen und dessen sündigem Verhängnis.

Anders gesagt: Der Schlüssel (Pascal spricht vom «Mysterium») der theologischen Anthropologie liegt in der Lehre von der Sünde. Auch hier entsteht ein Echo auf die reformatorische Auffassung der Theologie: Das sei hier betont, obschon es Pascal sehr unangenehm wäre, denn er hat solche Bezüge immer strikte abgelehnt, um die Katholizität seines Jansenismus zu wahren! Aber auch bei Luther ist das subiectum der Theologie als cognitio Dei et hominis «der schuldige und verlorene Mensch und der rechtfertigende oder rettende Gott». ${ }^{6}$ Und ganz im Sinne von Pascal und Luther formuliert viel später auch Bultmann, wenn es ihm darum geht, Gott und Mensch miteinander in Verbindung zu bringen: «[...] der Widerspruch des Menschen gegen Gott ist der Anknüpfungspunkt für den Widerspruch Gottes gegen ihn. Die Sünde des Menschen ist der Anknüpfungspunkt für das widersprechende Wort von der Gnade.» ${ }^{7}$

Pascal sieht im Mysterium der Sünde die paradoxe Möglichkeit, die Unbegreiflichkeit des Menschen zu begreifen, obschon gerade diese Lehre der menschlichen Vernunft als radikal unbegreiflich erscheint. "Gewiss ist uns nichts stärker zuwider als diese Lehre. Und doch sind wir ohne dieses Mysterium, das unbegreiflichste von allen, uns selbst unbegreiflich.» (92) Gleich im Anschluss an diesen Satz kann Pascal diese Sünde als den Abgrund bezeichnen, der den Menschen zum unbegreiflichen Monstrum von Grösse und Elend werden lässt: «Die Verwicklung unseres Daseins zieht ihre geheimsten Falten und Windungen aus diesem Abgrund.» (ebd.)

Und für Pascal gilt ähnlich wie für Bultmann: «Dann kann man also nicht auf diesen oder jenen Anknüpfungspunkt im Menschen, in seinem Geistesleben, in seiner Geschichte hinweisen. Vielmehr: der Mensch in seiner Existenz, als ganzer, ist der Anknüpfungspunkt. ${ }^{8}$ Mit den Begriffen Pascals: Im Zeichen des Mysteriums der Sünde ist der Mensch der Abgrund. Und wie Büchner sagte: Es schwindelt einem, wenn man hinabsieht ...

Aber aus diesem Abgrund heraus betet auch der Psalmist, wenn es heisst: «De profundis clamavi ad te Domine», eine Psalmstelle, die, wie auch andere vergleichbare, von Pascal öfters zitiert wird.

\footnotetext{
6 «[...] ut proprie sit subiectum Theologiae homo reus et perditus et deus iustificans vel salvator» (s. oben Anm. 1).

7 R. Bultmann, Anknüpfung und Widerspruch, in: ders., Glauben und Verstehen II, Tübingen ${ }^{5} 1968$, 117-132; hier 120 (Kursivsetzungen vom Autor).

8 Ebd., 120 f (Kursivsetzungen vom Autor).
} 


\section{Abgrund und Zerstreuung}

«Und ich widerspreche ihm immer. Bis er begreift, dass er ein unbegreifliches Monstrum ist.» So hiess es in der Pensée 130. Es lässt sich leicht nachvollziehen, dass diese paradoxe Selbsterkenntnis dem Menschen eher unlieb ist. Er wird versuchen, ihr partout zu entfliehen. Und damit sind wir bei einem weiteren wichtigen Thema von Pascals Anthropologie, dem er viel Aufmerksamkeit schenkt, dem Phänomen der Zerstreuung. ${ }^{9}$ Diese beobachtet und analysiert er in seiner Umwelt, etwa wenn der königliche Hof auf die Jagd geht oder wenn in den Pariser Salons mondäne Gespräche geführt werden. «Da die Menschen Tod, Elend und Unwissenheit nicht heilen konnten, sind sie, um sich glücklich zu machen, auf den Einfall gekommen, nicht daran zu denken.» ${ }^{10}$ Die Geschäftigkeit, mit der die Menschen ihr Leben füllen, indem sie jagen, spielen, Kriege führen, Geschäfte machen - Vorträge halten, Kongresse besuchen, Bücher schreiben usw. -, wird somit kritisch hinterfragt aufgrund der Einsicht, «dass das ganze Unglück der Menschen aus einem einzigen Umstand herrühre, nämlich, dass sie nicht ruhig in einem Zimmer bleiben können» (95).

Wenn die Tätigkeiten des Menschen allein dazu dienen, dem schwindelerregenden Abgrund von Grösse und Elend zu entgehen, besteht die Gefahr, dass genau das eintrifft, was man vermeiden wollte: "Wir eilen sorglos dem Abgrund entgegen, nachdem wir etwas vor uns aufgerichtet haben, um uns davon abzuhalten, ihn zu sehen.» ${ }^{11}$ Pascal kann diese Gefahr auch unmittelbar in der Spannung von Grösse und Elend zum Ausdruck bringen: Indem der Mensch nur das eine sein will: Grösse, die ihm passt, und das andere, das Elend, abtut als unpassend, unwichtig, unzeitgemäss, kann geschehen, dass genau dieses andere eintritt. «Der Mensch ist weder Engel noch Tier, und das Unglück will, dass wer Engel sein will, zum Tier wird.»"

Der Gefahr, in den Abgrund zu fallen, ihm zum Opfer zu werden, kann der Mensch nur entgehen, indem er sich diesen unbegreiflichen Abgrund im Hören auf Gott offenbaren lässt und sich ihm stellt, ihn als seine "véritable condition" wahr- und annimmt, indem er aus

\footnotetext{
9 Ein ganzes Kapitel der von Pascal geordneten Pensées, das Kapitel 8, ist diesem Thema gewidmet: vgl. Pascal, Gedanken (s. Anm. 3), 93-104.

${ }^{10}$ Ebd., 94 (Pensée 133; leicht korrigierte Übersetzung).

11 Ebd., 120 (Pensée 166).

12 Ebd., 378 (Pensée 678; korrigierte Übersetzung).
} 
dessen Tiefe heraus zu Gott ruft, zum Gott Abrahams, Isaaks und Jakobs, und nicht zum Gott der Philosophen und der Gelehrten. ${ }^{13}$

\section{Schlussbemerkung}

«Wir lesen, was dasteht. Aber was wir lesen, steht nicht einfach da. Sondern: dazwischen. Wo aber ist «zwischen`? Orten und dingfest machen lässt sich 'zwischen〉 nicht, auch nicht als dritter Ort zwischen scheinbar festen Ufern, ohne auszuschliessen, dass «zwischen〉 eine Funktion von Bewegung ist, die somit stillgelegt würde.» ${ }^{14}$

Ähnliches gilt wohl vom "zwischen» in meiner Überschrift:Auch das «zwischen Fragezeichen und Ausrufezeichen» steht nicht einfach $\mathrm{da}$, als stillgelegter dritter Ort, sondern lässt sich nicht orten und dingfest machen, ist Funktion von unablässiger Bewegung. Deshalb: Von Herzen sei hier dem Sechzigjährigen, «zwischen Wörtern, Sätzen, Zeilen» und Zeichen, diese unablässige Bewegung gewünscht!-?

\footnotetext{
${ }^{13}$ Vgl. Pascals berühmtes Gedenkblatt (Mémorial); Pascal, Gedanken (s. Anm. 3), 484. ${ }^{14}$ Ch. L. Hart Nibbrig, Zwischen Wörtern, Sätzen, Zeilen: Lesen, in: A. Härter/E. A Kunz/H. Weidmann (Hg.), Dazwischen. Zum transitorischen Denken in Literaturund Kulturwissenschaft (FS J. Anderegg), Göttingen 2003, 23-27, 23.
} 